\title{
Stochastic Stability Criteria for Neutral Distributed Parameter Systems with Markovian Jump
}

\author{
Yanbo Li $\left(\mathbb{D},{ }^{1}\right.$ Chao-Yang Chen $\mathbb{D}^{2,3}$ and Chengqun Li ${ }^{1}$ \\ ${ }^{1}$ School of Information and Statistics, Guangxi University of Finance and Economics, Nanning 530001, China \\ ${ }^{2}$ School of Information and Electrical Engineering, Hunan University of Science and Technology, Xiangtan 411201, China \\ ${ }^{3}$ Center for Polymer Studies and Department of Physics, Boston University, Boston, MA 02215, USA
}

Correspondence should be addressed to Chao-Yang Chen; ouzk@163.com

Received 16 June 2019; Revised 16 January 2020; Accepted 20 January 2020; Published 11 February 2020

Academic Editor: Mohamed Boutayeb

Copyright () 2020 Yanbo Li et al. This is an open access article distributed under the Creative Commons Attribution License, which permits unrestricted use, distribution, and reproduction in any medium, provided the original work is properly cited.

This paper deals with the problem of stochastic stability for a class of neutral distributed parameter systems with Markovian jump. In this model, we only need to know the absolute maximum of the state transition probability on the principal diagonal line; other transition rates can be completely unknown. Based on calculating the weak infinitesimal generator and combining Poincare inequality and Green formula, a stochastic stability criterion is given in terms of a set of linear matrix inequalities (LMIs) by the Schur complement lemma. Because of the existence of the neutral term, we need to construct Lyapunov functionals showing more complexity to handle the cross terms involving the Laplace operator. Finally, a numerical example is provided to support the validity of the mathematical results.

\section{Introduction}

Time-delay models are popular in all kinds of fields such as demography, biology, economics, and chemistry. Neutral systems as a special type of time-delay systems are often encountered because these systems have a wider application value than the general time-delay systems in many dynamical systems such as bioengineering systems, dynamic systems of offshore platform, and dynamic economic models. Hence, there are so many investigations about timedelay systems [1-7]. As we all know, the systems inevitably receive the impact of sudden changes in the environment, abrupt failure of components, unexpected changes in system parameters, and so on. These random diversifications usually follow the law of Markov jump. These systems are called Markovian jump systems. Markovian jump systems spur investigators' consuming interest $[8,9]$.

The stability and performance of stochastic systems are quite different from those of deterministic systems [10-12]. More recently, neutral-type Markovian jump systems have aspirated considerable attention. All kinds of analysis methods have been used to discover the stochastic stability criteria of neutral-type Markovian jump systems such as Lyapunov-Krasovskii functional approach, reciprocally convex combination inequality method, and stochastic analysis theory in [13-16]. For less conservative results, the delay-dependent stability has been discussed in [17-20]. Other control methods have also been extensively and thoroughly studied such as robust delay-dependent $H_{\infty}$ control [21, 22], nonfragile control [23-25], sliding mode control $[26,27], H_{\infty}$ sliding mode control [28], and the references therein.

The transition rates in many references mentioned above have been supposed to be completely known. But it is very hard to acquire the accurate transfer probability, and even if the exact transfer probability can be obtained, the cost is also very huge. So the study of Markovian jump systems with general unknown transition rates [29-50] has appealed to a great many scholars. Stability, stabilization, and robust control of Markovian jump systems with partially unknown transition have been reported in [29-33]. Stability analysis for neutral Markovian jump systems with partially unknown transition probabilities has been proposed in [34, 35]. Kao et al. and Yang et al. [36, 37] have settled the delay-dependent stability for Markovian jump systems with partially unknown 
transition probabilities and Markovian jump neutral stochastic systems with general unknown transition rates, respectively. Singular Markovian jump systems with general incomplete transition probabilities have been presented in [38-40]. Finite-time stochastic stability and control of Markovian jump systems with general incomplete transition probabilities have been discussed in [41-44]. Stabilization of discrete-time Markovian jump systems with partially unknown transition probabilities was probed in [45].

In parallel, many researchers have extensively studied the time-delay distributed parameter systems [46-54]. Three main approaches of time-delay distributed parameter systems are semigroup theory [47], matrix norm theory [48], and linear matrix inequality theory (LMI) [49]. The semigroup method cannot guarantee the system to be of a fine dynamic character and performance index undergoing two transformations. It is not easy to apply the results to practical problems by matrix norm. The problem of exponential stability and stabilization [50], sliding mode control [51], and feedback control [52] has been proposed in terms of the linear matrix inequality approach. However, less attention has been paid to the distributed parameter systems with Markovian jump, especially neutral distributed parameter systems with Markovian jump which requires a lot of research to be performed.

Based on previous discussions, we are considering the problem of stochastic stability of a class of Markovian jumping neutral distributed parameter systems in this paper. The linear matrix inequality approach together with the Lyapunov functional method is employed to develop stochastic stability criteria for the described systems. The results are given in a group of linear matrix inequalities (LMIs).

\section{Problem Formulation and Preliminaries}

Consider the neutral distributed parameter systems with Markovian jump of the following form:

$$
\begin{aligned}
\frac{\partial}{\partial t}[W(x, t)-C(r(t)) W(x, t-\sigma)]= & D(r(t)) \Delta W(x, t) \\
& +A(r(t)) W(x, t) \\
& +A_{1}(r(t)) W(x, t-\tau),
\end{aligned}
$$

where $(x, t) \in \Omega \times R_{+}, \Omega=\{x,\|x\|<l<+\infty\} \subset R^{m}$ is the bounded domain with smooth boundary $\partial \Omega$, and mes $\Omega>0$. Also,

$$
\nabla W(x, t)=\operatorname{col}\left(\nabla w_{1}(x, t), \nabla w_{2}(x, t), \ldots, \nabla w_{n}(x, t)\right),
$$

where $\nabla=\left(\left(\partial / \partial x_{1}\right),\left(\partial / \partial x_{2}\right), \ldots,\left(\partial / \partial x_{m}\right)\right)$ is the gradient operator.

$W(x, t)=\operatorname{col}\left(w_{1}(x, t), w_{2}(x, t), \ldots, w_{n}(x, t)\right) \in R^{n}$ is the state function, and $\Delta=\sum_{k=1}^{m} \partial^{2} / \partial x_{k}^{2}$ is the Laplace operator on $\Omega$.

The initial value and boundary value conditions satisfy

$$
\begin{aligned}
W(x, t) & =0,(x, t) \in \partial \Omega \times[-\gamma,+\infty), \\
W(x, t) & =\psi(x, t),(x, t) \in \Omega \times[-\gamma, 0], \\
\frac{\partial W(x, t)}{\partial n} & =0,(x, t) \in \partial \Omega \times[-\gamma,+\infty),
\end{aligned}
$$

where $\gamma=\max \{\sigma, \tau\}$ and $\sigma>0$ and $\tau>0$ are constants. $n$ is the unit outward normal vector of $\partial \Omega$, and $\psi(x, t)$ is the smooth function. $\tau>0, \sigma>0$, and $D(r(t))>0$ are constants; $A(r(t)), C(r(t))$, and $A_{1}(r(t))$ are constant matrices.

Let $\{r(t), t \geq 0\}$ be a right-continuous Markov process and take values in a finite set $F=\{1,2, \ldots, N\}$ with transition probability matrix $\Pi=\left(\pi_{i j}\right)$; the mode transition probabilities are defined as follows:

$$
P_{r}(r(t+h)=j \mid r(h)=i)= \begin{cases}\pi_{i j} h+o(h), & i \neq j, \\ 1+\pi_{i i} h+o(h), & i=j\end{cases}
$$

where $h>0$ and $\lim _{\delta \longrightarrow 0}(o(h) / h)=0 . \pi_{i j} \geq 0, i \neq j$ donates the transition rate from mode $i$ to mode $j$ in the time interval $h$ and $\pi_{i i}=-\sum_{j=1, j \neq i}^{N} \pi_{i j}$. For each $r(t)=i \in F$, let $A(r$ $(t))=A_{i}, A_{1}(r(t))=A_{1 i}, D(r(t))=D_{i}$, and $C(r(t))=C_{i}$. Then, we can represent system (1) in the following form:

$$
\begin{aligned}
\frac{\partial}{\partial t}\left[W(x, t)-C_{i} W(x, t-\sigma)\right]= & D_{i} \Delta W(x, t)+A_{i} W(x, t) \\
& +A_{1 i} W(x, t-\tau) .
\end{aligned}
$$

Lemma 1 (see [53] (Green formula)). Let $\Omega \subset R^{n}$ be the bounded domain with smooth boundary $\partial \Omega, n$ is the unit outward normal vector of $\partial \Omega$, and $G \subset \Omega$ is the smooth subdomain. If $u, v \in C^{2}(\bar{G})$, then

$$
\int_{G} u \Delta v \mathrm{~d} x=\int_{\partial \Omega} u \frac{\partial v}{\partial n} \mathrm{~d} s-\int_{\Omega} \nabla u \nabla v \mathrm{~d} x
$$

where $\nabla$ is the Hamilton operator and $d s$ is the area element over the boundary region.

Lemma 2 (see [54] (Friedrichs's inequality)). Let $w \in C_{0}^{1}(\Omega)$ and $\Omega$ be included in the closed region $\Omega_{1}: 0 \leq x_{i} \leq l(i=1,2, \ldots, n)$. Then,

$$
\int_{\Omega} w^{2}(x) \mathrm{d} x \leq \int_{\Omega} \sum_{i=1}^{n}\left(\frac{\partial w}{\partial x}\right)^{2} \mathrm{~d} x=c \int_{\Omega}|\nabla w|^{2} \mathrm{~d} x
$$

where $c=l^{2} / n$.

Lemma 3 (see [55]). Let $V_{1}, V_{2}$, and $V_{3}$ be the real matrices and $V_{3}=V_{3}^{T}>0$; then, for an arbitrary given scalar $\alpha>0$, the following inequality holds:

$$
V_{2}^{\mathrm{T}} V_{1}+V_{1}^{\mathrm{T}} V_{2} \leq \alpha^{-1} V_{1}^{\mathrm{T}} V_{3}^{-1} V_{1}+\alpha V_{2}^{\mathrm{T}} V_{3} V_{2} .
$$




\section{Main Results}

Theorem 1. Given matrices $A_{i}, A_{1 i}$, and $C_{i}$, time-delay constants $\tau>0$ and $\sigma>0$ and constant $D_{i}>0$, the neutral distributed parameter systems with Markovian jump (5) is stochastically stable. If there exist positive symmetric matrices $P_{i}, Q_{i}, M, N, R$, and $Z$ and positive scalars $\alpha_{i}$, such that for any Markovian jump mode $i \in F$, the following linear matrix inequalities (LMIs) hold:

$$
\Theta=\left(\begin{array}{cccccc}
\Pi_{1} & 0 & -A_{i}^{\mathrm{T}} C_{i} & 0 & 0 & 0 \\
0 & -P_{i} & -A_{1 i}^{\mathrm{T}} C_{i} & 0 & 0 & 0 \\
D_{i} C_{i}^{\mathrm{T}} & -\alpha_{i} I
\end{array}\right)<0,
$$

where $\beta=\max \left\{\left|\pi_{i i}\right|, i \in F\right\}, \Pi_{1}=A_{i}^{\mathrm{T}}+A_{i}+P_{i}+Q_{i}+\beta \tau R+$ $\beta \sigma Z, \Pi_{2}=M+N+A_{i}+A_{i}^{\mathrm{T}}-2 D_{i} I$,

$$
\begin{aligned}
& P_{i}<R, \\
& Q_{i}<Z .
\end{aligned}
$$

Proof. For system (5), we construct the following stochastic Lyapunov functional:

$$
V_{i}(t, W(x, t))=\sum_{n=1}^{9} V_{\text {in }},
$$

where

$$
\begin{aligned}
V_{1 i}= & \int_{\Omega} Y^{\mathrm{T}}(x, t) Y(x, t) \mathrm{d} x, \quad \text { where } Y(x, t)=W(x, t) \\
& -C_{i} W(x, t-\sigma), \\
V_{i 2}= & \int_{\Omega} \int_{t-\tau}^{t} W^{\mathrm{T}}(x, \theta) P_{i} W(x, \theta) \mathrm{d} \theta \mathrm{d} x, \\
V_{i 3}= & \int_{\Omega} \int_{t-\sigma}^{t} W^{\mathrm{T}}(x, \theta) Q_{i} W(x, \theta) \mathrm{d} \theta \mathrm{d} x, \\
V_{i 4}= & \int_{\Omega} \int_{t-\tau}^{t}\left(\nabla W^{\mathrm{T}}(x, \theta)\right) M\left(\nabla W^{\mathrm{T}}(x, \theta)\right)^{\mathrm{T}} \mathrm{d} \theta \mathrm{d} x, \\
V_{i 5}= & \int_{\Omega} \int_{t-\sigma}^{t}\left(\nabla W^{\mathrm{T}}(x, \theta)\right) N\left(\nabla W^{\mathrm{T}}(x, \theta)\right)^{\mathrm{T}} \mathrm{d} \theta \mathrm{d} x, \\
V_{i 6}= & \int_{\Omega}\left(\nabla Y^{\mathrm{T}}(x, \theta)\right)\left(\nabla Y^{\mathrm{T}}(x, \theta)\right)^{\mathrm{T}} \mathrm{d} x, \\
V_{i 7}= & \alpha_{i} \int_{\Omega} \int_{t-\sigma}^{t}(\Delta W(x, \theta))^{\mathrm{T}} \Delta W(x, \theta) \mathrm{d} \theta \mathrm{d} x, \\
V_{i 8}= & \beta_{i} \int_{\Omega} \int_{-\tau}^{0} \int_{t+s}^{t} W^{\mathrm{T}}(x, \theta) R W(x, \theta) \mathrm{d} \theta \mathrm{d} s \mathrm{~d} x, \\
V_{i 9}= & \beta_{i} \int_{\Omega} \int_{-\sigma}^{0} \int_{t+s}^{t} W^{\mathrm{T}}(x, \theta) Z W(x, \theta) \mathrm{d} \theta \mathrm{d} s \mathrm{~d} x .
\end{aligned}
$$

Let $L$ be the weak infinitesimal generator; then, we calculate

$$
L V_{i}(t, W(x, t))=\sum_{n=1}^{9} L V_{\text {in }}
$$

where

$$
\begin{aligned}
L V_{1 i}= & 2 \int_{\Omega} Y^{\mathrm{T}}(x, t) \frac{\partial Y(x, t)}{\partial t} \mathrm{~d} x \\
= & 2 D_{i} \int_{\Omega} W^{\mathrm{T}}(x, t) \Delta W(x, t) \mathrm{d} x \\
& +\int_{\Omega} W^{\mathrm{T}}(x, t)\left(A_{i}^{\mathrm{T}}+A_{i}\right) W(x, t) \mathrm{d} x \\
& +2 \int_{\Omega} W^{\mathrm{T}}(x, t) A_{1 i} W(x, t-\tau) \mathrm{d} x \\
& -2 D_{i} \int_{\Omega} W^{\mathrm{T}}(x, t-\sigma) C_{i}^{\mathrm{T}} \Delta W(x, t) \mathrm{d} x \\
& -2 \int_{\Omega} W^{\mathrm{T}}(x, t-\sigma) C_{i}^{\mathrm{T}} A_{i} W(x, t) \mathrm{d} x \\
& -2 \int_{\Omega} W^{\mathrm{T}}(x, t-\sigma) C_{i}^{\mathrm{T}} A_{1 i} W(x, t-\tau) \mathrm{d} x .
\end{aligned}
$$

Applying Lemma 1 and Lemma 2,

$$
\begin{aligned}
2 D_{i} \int_{\Omega} W^{\mathrm{T}}(x, t) \Delta W(x, t) \mathrm{d} x= & \sum_{k=1}^{n} \sum_{l=1}^{n}\left[\int_{\partial \Omega} w_{k}(x, t) \frac{\partial w_{l}(x, t)}{\partial n} \mathrm{~d} s\right. \\
& \left.-\int_{\Omega} \nabla w_{k}(x, t) \nabla w_{l}(x, t) \mathrm{d} x\right] \\
= & -\sum_{k=1}^{n} \sum_{l=1}^{n} \int_{\Omega} \nabla w_{k}(x, t) \nabla w_{l}(x, t) \mathrm{d} x \\
= & -2 D_{i} \int_{\Omega} \nabla W^{\mathrm{T}}(x, t)\left(\nabla W^{\mathrm{T}}(x, t)\right)^{\mathrm{T}} \mathrm{d} x,
\end{aligned}
$$

$$
-2 D_{i} \int_{\Omega} W^{\mathrm{T}}(x, t-\sigma) C_{i}^{\mathrm{T}} \Delta W(x, t) \mathrm{d} x
$$$$
=-2 D_{i} \int_{\Omega} \sum_{l=1}^{n} \sum_{m=1}^{n} w_{m}(x, t-\sigma) c_{m l} \nabla \cdot\left(\nabla w_{l}(x, t)\right) \mathrm{d} x
$$$$
=2 D_{i} \sum_{l=1}^{n} \sum_{m=1}^{n} \int_{\Omega} \nabla w_{m}(x, t-\sigma) c_{m l} \cdot \nabla w_{l}(x, t) \mathrm{d} x
$$$$
=2 D_{i} \sum_{l=1}^{n} \sum_{m=1}^{n} \sum_{k=1}^{m} \int_{\Omega} \frac{\partial w_{m}(x, t-\sigma)}{\partial x_{k}} c_{m l} \frac{\partial w_{l}(x, t)}{\partial x_{k}} \mathrm{~d} x
$$$$
=2 D_{i} \int_{\Omega} \nabla W^{\mathrm{T}}(x, t-\sigma) C_{i}\left(\nabla W^{\mathrm{T}}(x, t)\right)^{\mathrm{T}} \mathrm{d} x .
$$

Substituting (16) and (17) into (15), we have 


$$
\begin{aligned}
& L V_{1 i}=-2 D_{i} \int_{\Omega} \nabla W^{\mathrm{T}}(x, t)\left(\nabla W^{\mathrm{T}}(x, t)\right)^{\mathrm{T}} \mathrm{d} x \\
& +\int_{\Omega} W^{\mathrm{T}}(x, t)\left(A_{i}^{\mathrm{T}}+A_{i}\right) W(x, t) \mathrm{d} x \\
& +2 \int_{\Omega} W^{\mathrm{T}}(x, t) A_{1 i} W(x, t-\tau) \mathrm{d} x \\
& +2 D_{i} \int_{\Omega} \nabla W^{\mathrm{T}}(x, t-\sigma) C_{i}^{\mathrm{T}}\left(\nabla W^{\mathrm{T}}(x, t)\right)^{\mathrm{T}} \mathrm{d} x \\
& -2 \int_{\Omega} W^{\mathrm{T}}(x, t-\sigma) C_{i}^{\mathrm{T}} A_{i} W(x, t) \mathrm{d} x \\
& -2 \int_{\Omega} W^{\mathrm{T}}(x, t-\sigma) C_{i}^{\mathrm{T}} A_{1 i} W(x, t-\tau) \mathrm{d} x, \\
& L V_{i 2}(t, W(x, t))=\int_{\Omega} W^{\mathrm{T}}(x, t) P_{i} W(x, t) \mathrm{d} x \\
& -\int_{\Omega} W^{\mathrm{T}}(x, t-\tau) P_{i} W(x, t-\tau) \mathrm{d} x \\
& +\int_{\Omega} \int_{t-\tau}^{t} W^{\mathrm{T}}(x, \theta)\left(\sum_{j=1}^{N} \pi_{i j} P_{j}\right) W \\
& \cdot(x, \theta) \mathrm{d} \theta \mathrm{d} x \text {. }
\end{aligned}
$$

Noticing $\pi_{i j}>0(i \neq j)$ and combining $P_{j}<R$ and $\beta=\max \left\{\left|\pi_{i i}\right|, i \in F\right\}$, we derive the following inequality:

$$
\begin{aligned}
& \int_{\Omega} \int_{t-\tau}^{t} W^{\mathrm{T}}(x, \theta)\left(\sum_{j=1}^{N} \pi_{i j} P_{j}\right) W(x, \theta) \mathrm{d} \theta \mathrm{d} x \\
& \leq \int_{\Omega} \int_{t-\tau}^{t} W^{\mathrm{T}}(x, \theta)\left(\sum_{j=1, i \neq j}^{N} \pi_{i j} P_{j}\right) W(x, \theta) \mathrm{d} \theta \mathrm{d} x \\
& =-\pi_{i i} \int_{\Omega} \int_{t-\tau}^{t} W^{\mathrm{T}}(x, \theta) P_{j} W(x, \theta) \mathrm{d} \theta \mathrm{d} x \\
& \leq \beta \int_{\Omega} \int_{t-\tau}^{t} W^{\mathrm{T}}(x, \theta) P_{j} W(x, \theta) \mathrm{d} \theta \mathrm{d} x \\
& \leq \beta \int_{\Omega} \int_{t-\tau}^{t} W^{\mathrm{T}}(x, \theta) R W(x, \theta) \mathrm{d} \theta \mathrm{d} x .
\end{aligned}
$$

Then,

$$
\begin{aligned}
L V_{i 2}(t, W(x, t)) \leq & \int_{\Omega} W^{\mathrm{T}}(x, t) P_{i} W(x, t) \mathrm{d} x \\
& -\int_{\Omega} W^{\mathrm{T}}(x, t-\tau) P_{i} W(x, t-\tau) \mathrm{d} x \\
& +\beta \int_{\Omega} \int_{t-\tau}^{t} W^{\mathrm{T}}(x, \theta) R W(x, \theta) \mathrm{d} \theta \mathrm{d} x .
\end{aligned}
$$

For the same reason, we can also obtain

$$
\begin{aligned}
& \int_{\Omega} \int_{t-\sigma}^{t} W^{\mathrm{T}}(x, \theta)\left(\sum_{j=1}^{N} \pi_{i j} Q_{j}\right) W(x, \theta) \mathrm{d} \theta \mathrm{d} x \\
& \leq \beta \int_{\Omega} \int_{t-\sigma}^{t} W^{\mathrm{T}}(x, \theta) Q_{j} W(x, \theta) \mathrm{d} \theta \mathrm{d} x \\
& \leq \beta \int_{\Omega} \int_{t-\sigma}^{t} W^{\mathrm{T}}(x, \theta) Z W(x, \theta) \mathrm{d} \theta \mathrm{d} x .
\end{aligned}
$$

$$
\begin{aligned}
& \text { So } \\
& \begin{aligned}
L V_{i 3}(t, W(x, t))= & \int_{\Omega} W^{\mathrm{T}}(x, t) Q_{i} W(x, t) \mathrm{d} x \\
& -\int_{\Omega} W^{\mathrm{T}}(x, t-\sigma) Q_{i} W(x, t-\sigma) \mathrm{d} x \\
& +\int_{\Omega} \int_{t-\sigma}^{t} W^{\mathrm{T}}(x, \theta)\left(\sum_{j=1}^{N} \pi_{i j} Q_{j}\right) \\
& \cdot W(x, \theta) \mathrm{d} \theta \mathrm{d} x \\
\leq & \int_{\Omega} W^{\mathrm{T}}(x, t) Q_{i} W(x, t) \mathrm{d} x \\
& -\int_{\Omega} W^{\mathrm{T}}(x, t-\sigma) Q_{i} W(x, t-\sigma) \mathrm{d} x \\
& +\beta \int_{\Omega} \int_{t-\sigma}^{t} W^{\mathrm{T}}(x, \theta) Z W(x, \theta) \mathrm{d} \theta \mathrm{d} x,
\end{aligned}
\end{aligned}
$$

$$
\begin{aligned}
L V_{i 4}= & \int_{\Omega} \nabla W^{\mathrm{T}}(x, t) M\left(\nabla W^{\mathrm{T}}(x, t)\right)^{\mathrm{T}} \mathrm{d} x \\
& -\int_{\Omega} \nabla W^{\mathrm{T}}(x, t-\tau) M\left(\nabla W^{\mathrm{T}}(x, t-\tau)\right)^{\mathrm{T}} \mathrm{d} x, \\
L V_{i 5}= & \int_{\Omega} \nabla W^{\mathrm{T}}(x, t) N\left(\nabla W^{\mathrm{T}}(x, t)\right)^{\mathrm{T}} \mathrm{d} x \\
& -\int_{\Omega} \nabla W^{\mathrm{T}}(x, t-\sigma) N\left(\nabla W^{\mathrm{T}}(x, t-\sigma)\right)^{\mathrm{T}} \mathrm{d} x, \\
L V_{i 6}= & 2 \int_{\Omega}\left(\nabla \frac{\partial Y^{\mathrm{T}}(x, t)}{\partial t}\right)^{\mathrm{T}}\left(\nabla Y^{\mathrm{T}}(x, t)\right)^{\mathrm{T}} \mathrm{d} x \\
= & -2 \int_{\Omega} \frac{\partial Y^{\mathrm{T}}(x, t)}{\partial t}\left(\Delta Y^{\mathrm{T}}(x, t)\right)^{\mathrm{T}} \mathrm{d} x \\
= & -2 D_{i} \int_{\Omega}(\Delta W(x, t))^{\mathrm{T}} \Delta W(x, t) \mathrm{d} x \\
& +2 D_{i} \int_{\Omega}(\Delta W(x, t))^{\mathrm{T}} C_{i} \Delta W(x, t-\sigma) \mathrm{d} x \\
& -2 \int_{\Omega} W^{\mathrm{T}}(x, t) A_{i}^{\mathrm{T}} \Delta W(x, t) \mathrm{d} x \\
& -2 \int_{\Omega} W^{\mathrm{T}}(x, t-\tau) A_{1 i}^{\mathrm{T}} \Delta W(x, t) \mathrm{d} x \\
& +2 \int_{\Omega} W^{\mathrm{T}}(x, t) A_{i}^{\mathrm{T}} C_{i} \Delta W(x, t-\sigma) \mathrm{d} x \\
& +2 \int_{\Omega}^{\mathrm{T}}(x, t-\tau) A_{1 i}^{\mathrm{T}} C_{i} \Delta W(x, t-\sigma) \mathrm{d} x . \\
& \\
& \\
&
\end{aligned}
$$



holds:

Through applying Lemma 3, the following inequality Taking advantage of Lemma 1 and Lemma 2, we obtain

$$
\begin{aligned}
& 2 D_{i} \int_{\Omega}(\Delta W(x, t))^{\mathrm{T}} C_{i} \Delta W(x, t-\sigma) \mathrm{d} x \\
& \leq \alpha_{i}^{-1} \int_{\Omega}(\Delta W(x, t))^{\mathrm{T}} D_{i}^{2} C_{i} C_{i}^{\mathrm{T}} \Delta W(x, t) \mathrm{d} x \\
& \quad+\alpha_{i} \int_{\Omega}(\Delta W(x, t-\sigma))^{\mathrm{T}}(\Delta W(x, t-\sigma)) \mathrm{d} x .
\end{aligned}
$$

$$
\begin{gathered}
-2 \int_{\Omega} W^{\mathrm{T}}(x, t) A_{i}^{\mathrm{T}} \Delta W(x, t) \mathrm{d} x=2 \int_{\Omega}\left(\nabla W^{\mathrm{T}}(x, t)\right) A_{i}\left(\nabla W^{\mathrm{T}}(x, t)\right)^{\mathrm{T}} \mathrm{d} x, \\
2 \int_{\Omega} W^{\mathrm{T}}(x, t) A_{i}^{\mathrm{T}} C_{i} \Delta W(x, t-\sigma) \mathrm{d} x=-2 \int_{\Omega}\left(\nabla W^{\mathrm{T}}(x, t)\right) C_{i}^{\mathrm{T}} A_{i}\left(\nabla W^{\mathrm{T}}(x, t-\sigma)\right)^{\mathrm{T}} \mathrm{d} x, \\
-2 \int_{\Omega} W^{\mathrm{T}}(x, t-\tau) A_{1 i}^{\mathrm{T}} \Delta W(x, t) \mathrm{d} x=2 \int_{\Omega}\left(\nabla W^{\mathrm{T}}(x, t-\tau)\right) A_{1 i}\left(\nabla W^{\mathrm{T}}(x, t)\right)^{\mathrm{T}} \mathrm{d} x, \\
2 \int_{\Omega} W^{\mathrm{T}}(x, t-\tau) A_{1 i}^{\mathrm{T}} C_{i} \Delta W(x, t-\sigma) \mathrm{d} x=-2 \int_{\Omega}\left(\nabla W^{\mathrm{T}}(x, t-\tau)\right) C_{i}^{\mathrm{T}} A_{1 i}\left(\nabla W^{\mathrm{T}}(x, t-\sigma)\right)^{\mathrm{T}} \mathrm{d} x .
\end{gathered}
$$

Combining (26)-(31) together yields

$$
\begin{aligned}
& L V_{i 6} \leq \int_{\Omega}(\Delta W(x, t))^{\mathrm{T}}\left(\alpha_{i}^{-1} D_{i}^{2} C_{i} C_{i}^{\mathrm{T}}-2 D_{i} I\right) \Delta W(x, t) \mathrm{d} x \\
& +\alpha_{i} \int_{\Omega}(\Delta W(x, t-\sigma))^{\mathrm{T}}(\Delta W(x, t-\sigma)) \mathrm{d} x \\
& +2 \int_{\Omega}\left(\nabla W^{\mathrm{T}}(x, t)\right) A_{i}\left(\nabla W^{\mathrm{T}}(x, t)\right)^{\mathrm{T}} \mathrm{d} x \\
& +2 \int_{\Omega}\left(\nabla W^{\mathrm{T}}(x, t-\tau)\right) A_{1 i}\left(\nabla W^{\mathrm{T}}(x, t)\right)^{\mathrm{T}} \mathrm{d} x \\
& -2 \int_{\Omega}\left(\nabla W^{\mathrm{T}}(x, t)\right) C_{i}^{\mathrm{T}} A_{i}\left(\nabla W^{\mathrm{T}}(x, t-\sigma)\right)^{\mathrm{T}} \mathrm{d} x \\
& -2 \int_{\Omega}\left(\nabla W^{\mathrm{T}}(x, t-\tau)\right) C_{i}^{\mathrm{T}} A_{1 i}\left(\nabla W^{\mathrm{T}}(x, t-\sigma)\right)^{\mathrm{T}} \mathrm{d} x, \\
& L V_{i 7}=\alpha_{i} \int_{\Omega}(\Delta W(x, t))^{\mathrm{T}} \Delta W(x, t) \mathrm{d} x \\
& -\alpha_{i} \int_{\Omega}(\Delta W(x, t-\sigma))^{\mathrm{T}}(\Delta W(x, t-\sigma)) \mathrm{d} x, \\
& L V_{i 8}=\beta \tau \int_{\Omega} W^{\mathrm{T}}(x, t) R W(x, t) \mathrm{d} x \\
& -\beta \int_{\Omega} \int_{t-\tau}^{t} W^{\mathrm{T}}(x, \theta) R W(x, \theta) \mathrm{d} \theta \mathrm{d} x, \\
& L V_{i 9}=\beta \sigma \int_{\Omega} W^{\mathrm{T}}(x, t) Z W(x, t) \mathrm{d} x \\
& -\beta \int_{\Omega} \int_{t-\sigma}^{t} W^{\mathrm{T}}(x, \theta) Z W(x, \theta) \mathrm{d} \theta \mathrm{d} x .
\end{aligned}
$$

Synthesizing (18), (21), (23)-(25), and (32)-(35), the following inequality holds:

$$
L V(t, W(x, t)) \leq \int_{\Omega}(\Delta W(x, t))^{\mathrm{T}}\left(\alpha_{i} I+\alpha_{i}^{-1} D_{i}^{2} C_{i} C_{i}^{\mathrm{T}}\right.
$$$$
\left.-2 D_{i} I\right) \Delta W(x, t) \mathrm{d} x
$$$$
+\int_{\Omega} W^{\mathrm{T}}(x, t) \Pi_{1} W(x, t) \mathrm{d} x
$$$$
+\int_{\Omega} \nabla W^{\mathrm{T}}(x, t) \Pi_{2}\left(\nabla W^{\mathrm{T}}(x, t)\right)^{\mathrm{T}} \mathrm{d} x
$$$$
-\int_{\Omega} W^{\mathrm{T}}(x, t-\tau) P_{i} W(t-\tau) \mathrm{d} x
$$$$
-\int_{\Omega} W^{\mathrm{T}}(x, t-\sigma) Q_{i} W(t-\sigma) \mathrm{d} x
$$$$
-\int_{\Omega} \nabla W^{\mathrm{T}}(x, t-\tau) M\left(\nabla W^{\mathrm{T}}(x, t-\tau)\right)^{\mathrm{T}} \mathrm{d} x
$$$$
-\int_{\Omega} \nabla W^{\mathrm{T}}(x, t-\sigma) N\left(\nabla W^{\mathrm{T}}(x, t-\sigma)\right)^{\mathrm{T}} \mathrm{d} x
$$$$
+2 \int_{\Omega} \nabla W^{\mathrm{T}}(x, t-\sigma)\left(D_{i} C_{i}^{\mathrm{T}}-A_{i}^{\mathrm{T}} C_{i}\right)
$$$$
\cdot\left(\nabla W^{\mathrm{T}}(x, t)\right)^{\mathrm{T}} \mathrm{d} x
$$$$
+2 \int_{\Omega}\left(\nabla W^{\mathrm{T}}(x, t-\tau)\right) A_{1 i}\left(\nabla W^{\mathrm{T}}(x, t)\right)^{\mathrm{T}} \mathrm{d} x
$$$$
-2 \int_{\Omega}\left(\nabla W^{\mathrm{T}}(x, t-\tau)\right) C_{i}^{\mathrm{T}} A_{1 i}
$$$$
\cdot\left(\nabla W^{\mathrm{T}}(x, t-\sigma)\right)^{\mathrm{T}} \mathrm{d} x
$$$$
-2 \int_{\Omega} W^{\mathrm{T}}(x, t-\sigma) C_{i}^{\mathrm{T}} A_{i} W(x, t) \mathrm{d} x
$$$$
-2 \int_{\Omega} W^{\mathrm{T}}(x, t-\sigma) C_{i}^{\mathrm{T}} A_{1 i} W(x, t-\tau) \mathrm{d} x .
$$ 
Select appropriate $\alpha_{i}>0$ such that $\alpha_{i} I+\alpha_{i}^{-1} D_{i}^{2}$ $C_{i} C_{i}^{\mathrm{T}}-2 D_{i} I<0$, then we transform it into (9) by the Schur complement lemma.

Set

$$
X(t)=\left(\begin{array}{c}
W(x, t) \\
W(x, t-\tau) \\
W(x, t-\sigma) \\
\left(\nabla W^{\mathrm{T}}(x, t)\right)^{\mathrm{T}} \\
\left(\nabla W^{\mathrm{T}}(x, t-\tau)\right)^{\mathrm{T}} \\
\left(\nabla W^{\mathrm{T}}(x, t-\sigma)\right)^{\mathrm{T}}
\end{array}\right) .
$$

Then,

$$
L V(x, W(x, t)) \leq \int_{\Omega} X^{\mathrm{T}}(t) \Theta X(t) \mathrm{d} x<0 .
$$

The proof is concluded.

Remark 1. Because of the simultaneous existence of neutral term and Markov jump, the stochastic stability of neutral distributed parameter systems with jump is given by finding the maximum value of absolute value on the main diagonal of the state transition probability matrix. Compared with the general time-delay system, the derivation process of the system is complex, but the conclusion is simple.

\section{Examples}

Consider a neutral distributed parameter system (5) with two Markovian jump modes and the parameters as follows:

$$
\begin{aligned}
A_{1} & =\left(\begin{array}{cc}
-1 & 0 \\
0 & -0.9
\end{array}\right), \\
A_{2} & =\left(\begin{array}{cc}
-1 & 0 \\
0 & -0.1
\end{array}\right), \\
A_{11} & =\left(\begin{array}{cc}
1 & 0.1 \\
-0.5 & 1
\end{array}\right), \\
A_{12} & =\left(\begin{array}{cc}
-1 & 2 \\
0 & -1
\end{array}\right), \\
C_{1} & =\left(\begin{array}{cc}
-0.3 & 0.5 \\
0 & -0.2
\end{array}\right), \\
C_{2} & =\left(\begin{array}{cc}
-0.2 & 0.3 \\
-0.1 & -1.6
\end{array}\right), \\
D_{1} & =D_{2}=1, \\
\tau & =0.1, \\
\sigma & =0.2 .
\end{aligned}
$$

The transition rate matrix is defined by

$$
\Pi=\left(\begin{array}{cc}
-1 & 1 \\
0.5 & -0.5
\end{array}\right) \text {. }
$$

Obviously, the absolute maximum of the principle diagonal line is 1 . We get feasible solutions by solving linear matrix inequalities (9)-(12) in Theorem 1 and obtain the following parameters:

$$
\begin{aligned}
M & =\left(\begin{array}{ll}
2.4183 & 0.3114 \\
0.3114 & 0.4770
\end{array}\right), \\
N & =\left(\begin{array}{ll}
0.6491 & 0.0022 \\
0.0022 & 1.1412
\end{array}\right), \\
P_{1} & =\left(\begin{array}{ll}
17.3237 & 1.3197 \\
1.3197 & 21.9271
\end{array}\right), \\
P_{2} & =\left(\begin{array}{ll}
35.0493 & 15.3697 \\
15.3697 & 80.9490
\end{array}\right), \\
Q_{1} & =\left(\begin{array}{cc}
0.0358 & -0.0829 \\
-0.0829 & 0.2264
\end{array}\right), \\
Q_{2} & =\left(\begin{array}{cc}
0.0844 & -0.1372 \\
-0.1372 & 0.5661
\end{array}\right), \\
R & =\left(\begin{array}{ll}
5.2693 & 0.2587 \\
0.2587 & 3.0645
\end{array}\right), \\
Z & =\left(\begin{array}{ll}
3.6731 & 0.2179 \\
0.2179 & 1.4507
\end{array}\right) .
\end{aligned}
$$

Obviously matrices $P_{1}, P_{2}, Q_{1}, Q_{2}, M, N, R$, and $Z$ are positive. The effectiveness of the method of Theorem 1 is illustrated.

\section{Conclusion}

First, we choose a set of appropriate Lyapunov stochastic functionals; some of them show more complexity in dealing with the neutral cross terms containing the Laplace operator. Then, by taking advantage of boundary conditions, Green formula, and Schur complement lemma, we obtain a sufficient condition for stochastic stability of the studied model in this paper via linear matrix inequalities. At last, a numerical example is given to prove the effectiveness of the proposed method.

\section{Data Availability}

The simulation data used to support the findings of this study are included within the article.

\section{Conflicts of Interest}

The author declares that there are no conflicts of interest regarding the publication of this paper.

\section{Acknowledgments}

This work was supported in part by the Guangxi Natural Science Foundation Program under Grant nos. 2016GXNSFAA380157 and 2019JJA110076 and the National Natural Science Foundation of China (61973110, 71961002, and 61503133). 


\section{References}

[1] Y. He, M. Wu, J.-H. She, and G.-P. Liu, "Delay-dependent robust stability criteria for uncertain neutral systems with mixed delays," Systems \& Control Letters, vol. 51, no. 1, pp. 57-65, 2004.

[2] G. I. B. and B. Mohamed, "Static output feedback stabilization with $H_{\infty}$ performance for linear discrete-time systems," IEEE Transactions on Automatic Control, vol. 50, no. 2, pp. 250254, 2005.

[3] R. Krishnasamy and P. Balasubramaniam, "Robust stability results for nonlinear Markovian jump systems with modedependent time-varying delays and randomly occurring uncertainties," Complexity, vol. 21, no. 6, pp. 50-60, 2016.

[4] Y. Liu, S. M. Lee, O. M. Kwon, and J. H. Park, "Delay-dependent exponential stability criteria for neutral systems with interval time-varying delays and nonlinear perturbations," Journal of the Franklin Institute, vol. 350, no. 10, pp. 33133327, 2013.

[5] J. Xia, J. H. Park, B. Zhang, and H. Shen, "Robust $H_{\infty}$ tracking control for uncertain Markovian jumping systems with interval time-varying delay," Complexity, vol. 21, no. 2, pp. 355-366, 2015.

[6] C. Y. Chen, Y. Tang, L. H. Wu et al., "Adaptive Neuralnetwork-based control for a class of nonlinear systems with unknown output disturbance and time delays," IEEE Access, vol. 7, pp. 7702-7716, 2019.

[7] Q. L. Han, "Stability criteria for a class of linear neutral systems with time-varying discrete and distributed delays," IMA Journal of Mathematical Control and Information, vol. 20, no. 4, pp. 371-386, 2018.

[8] S. Zhu, Y. Shen, and L. Liu, "Exponential stability of uncertain stochastic neural networks with markovian switching," Neural Processing Letters, vol. 32, no. 3, pp. 293-309, 2010.

[9] S. Zhu and Y. Shen, "Passivity analysis of stochastic delayed neural networks with Markovian switching," Neurocomputing, vol. 74, no. 10, pp. 1754-1761, 2011.

[10] C.-Y. Chen, W.-H. Gui, Z.-H. Guan, R.-L. Wang, and S.-W. Zhou, "Adaptive neural control for a class of stochastic nonlinear systems with unknown parameters, unknown nonlinear functions and stochastic disturbances," Neurocomputing, vol. 226, pp. 101-108, 2017.

[11] C. Y. Chen, W. H. Gui, L. H. Wu et al., "Tracking performance limitation of MIMO NCSs with multiple communication constraints," IEEE Transactions on Cybernetics, 2019.

[12] C.-Y. Chen, Z.-H. Guan, M. Chi, Y. Wu, R.-Q. Liao, and X.-W. Jiang, "Fundamental performance limitations of networked control systems with novel trade-off factors and constraint channels," Journal of the Franklin Institute, vol. 354, no. 7, pp. 3120-3133, 2017.

[13] H. Zhang, Z. Qiu, L. Xiong, and G. Jiang, "Stochastic stability analysis for neutral-type Markov jump neural networks with additive time-varying delays via a new reciprocally convex combination inequality," International Journal of Systems Science, vol. 50, no. 5, pp. 970-988, 2019.

[14] J.-Q. Qiu, J. Chen, P. Shi, and H.-J. Yang, "New stochastic robust stability criteria for time-varying delay neutral system with Markovian jump parameters," International Journal of Control, Automation and Systems, vol. 8, no. 2, pp. 418-424, 2010.

[15] Q. Zhu and J. Cao, "Stability analysis for stochastic neural networks of neutral type with both Markovian jump parameters and mixed time delays," Neurocomputing, vol. 73, no. 13-15, pp. 2671-2680, 2010.
[16] T. Ma and L. Li, "Stability analysis for uncertain neutral-type stochastic nonlinear systems with mixed time-varying delays," Journal of Inequalities and Applications, vol. 2019, no. 1, 153 pages, 2019.

[17] R. Manivannan, R. Samidurai, J. Cao, A. Alsaedi, and F. E. Alsaedi, "Delay-dependent stability criteria for neutraltype neural networks with interval time-varying delay signals under the effects of leakage delay," Advances in Difference Equations, vol. 2018, no. 1, p. 53, 2018.

[18] J. Xia, H. Park, and H. Zeng, "Improved delay-dependent robust stability analysis for neutral-type uncertain neural networks with markovian jumping parameters and timevarying delays," Neurocomputing, vol. 149, pp. 1198-1205, 2015.

[19] P.-L. Liu, "Further improvement on delay-derivative-dependent stochastic stability criteria for markovian jumping neutral-type interval time-varying delay systems with mixed delays," International Journal of Control, Automation and Systems, vol. 17, no. 8, pp. 1899-1910, 2019.

[20] J. Sun, G. P. Liu, and J. Chen, "Delay-dependent stability and stabilization of neutral time-delay systems," International Journal of Robust \& Nonlinear Control, vol. 19, no. 12, pp. 1364-1375, 2009.

[21] H. R. Karimi, "Robust delay-dependent $H_{\infty}$ control of uncertain time-delay systems with mixed neutral, discrete, and distributed time-delays and markovian switching parameters," IEEE Transactions on Circuits and Systems I: Regular Papers, vol. 58, no. 8, pp. 1910-1923, 2011.

[22] H. Lu, W. Zhou, C. Duan, and X. Qi, "Delay-dependent robust $H_{\infty}$ control for uncertain singular time-delay system with Markovian jumping parameters," Optimal Control Applications and Methods, vol. 34, no. 3, pp. 296-307, 2013.

[23] B. Jiang, Y. Kao, and C. Gao, "Non-fragile feedback control with $L_{2}$ gain performance of uncertain neutral-type stochastic Markovian jump systems," International Journal of Systems Science, vol. 48, no. 7, pp. 1496-1506, 2017.

[24] G. Chen, Y. Shen, and S. Zhu, "Non-fragile observer-based $H_{\infty}$ control for neutral stochastic hybrid systems with timevarying delay," Neural Computing and Applications, vol. 20, no. 8, pp. 1149-1158, 2011.

[25] M. Hua, H. Tan, J. Chen, and J. Fei, "Robust delay-rangedependent non-fragile $H_{\infty}$ filtering for uncertain neutral stochastic systems with Markovian switching and mode-dependent time delays," Journal of the Franklin Institute, vol. 352, no. 3, pp. 1318-1341, 2015.

[26] Y. Kao, J. Xie, C. Wang et al., "A sliding mode approach to $H_{\infty}$ non-fragile observer-based control design for uncertain Markovian neutral-type stochastic systems," Automatica, vol. 52, pp. 218-226, 2015.

[27] Z. Liu, C. Gao, H. Xiao, and Y. Kao, "Non-fragile observerbased $H_{\infty}$ control for uncertain neutral-type systems via sliding mod mode technique," Asian Journal of Control, vol. 19, no. 2, pp. 659-671, 2017.

[28] Y. G. Kao, C. H. Wang, J. Xie, H. R. Karimi, and W. Li, " $H_{\infty}$ sliding mode control for uncertain neutral-type stochastic Markovian jumping parameters," Information Sciences, vol. 314, pp. 200-211, 2015.

[29] L. Zhang and E.-K. Boukas, "Stability and stabilization of Markovian jump linear systems with partly unknown transition probabilities," Automatica, vol. 45, no. 2, pp. 463-468, 2009.

[30] B. Du, J. Lam, Y. Zou et al., "Stability and stabilization for Markovian jump time-delay systems with partially unknown transition rates," IEEE Transactions on Circuits and Systems I: Regular Papers, vol. 60, no. 2, pp. 341-351, 2013. 
[31] E. Tian, D. Yue, and G. Wei, "Robust control for Markovian jump systems with partially known transition probabilities and nonlinearities," Journal of the Franklin Institute, vol. 350, no. 8, pp. 2069-2083, 2013.

[32] K. Ramakrishnan and G. Ray, "Robust stability criterion for Markovian jump systems with nonlinear perturbations and mode- dependent time delays," International Journal of General Systems, vol. 41, no. 4, pp. 373-393, 2012.

[33] X. H. Liu and H. S. Xi, "Stability analysis for neutral delay Markovian jump systems with nonlinear perturbations and partially unknown transition rates," Advances in Mathematical Physics, vol. 2013, Article ID 101485, 20 pages, 2013.

[34] L. Xiong, J. Tian, and X. Liu, "Stability analysis for neutral Markovian jump systems with partially unknown transition probabilities," Journal of the Franklin Institute, vol. 349, no. 6, pp. 2193-2214, 2012.

[35] Y. Zhang, K. Lou, and Y. Ge, "New result on delay-dependent stability for Markovian jump time-delay systems with partial information on transition probabilities," IEEE/CAA Journal of Automatica Sinica, vol. 6, no. 6, 2019.

[36] Y. Kao, C. Wang, J. Xie et al., "New delay-dependent stability of Markovian jump neutral stochastic systems with general unknown transition rates," International Journal of Systems Science, vol. 47, no. 11, pp. 2499-2509, 2016.

[37] G. Yang, Y. Kao, B. Jiang et al., "Delay-dependent $H_{\infty}$ filtering for singular Markovian jump systems with general uncomplete transition probabilities," Applied Mathematics and Computation, vol. 294, pp. 195-215, 2017.

[38] H. S. Kim, "Delay-dependent stability analysis for singular Markovian jump systems with incomplete transition probabilities," Journal of the Franklin Institute, vol. 352, no. 1, pp. 236-247, 2015.

[39] G. Wang, J. Li, L. Li et al., "Robust finite-time $H_{\infty}$ control for uncertain singular stochastic Markovian jump systems via proportional differential control law," IET Control Theory \& Applications, vol. 8, no. 16, pp. 1625-1638, 2014.

[40] Y. Wei and W. X. Zheng, "Finite-time stochastic stabilization of Markovian jump non-linear quadratic systems with partially known transition probabilities," IET Control Theory \& Applications, vol. 8, no. 5, pp. 311-318, 2014.

[41] Z. Zuo, Y. Liu, Y. Wang et al., "Finite-time stochastic stability and stabilization of linear discrete-time Markovian jump systems with partly unknown transition probabilities," IET Control Theory \& Applications, vol. 6, no. 10, pp. 1522-1526, 2012.

[42] Z. Yan, J. H. Park, and W. Zhang, "Finite-time guaranteed cost control for Ito Stochastic Markovian jump systems with incomplete transition rates," International Journal of Robust and Nonlinear Control, vol. 27, no. 1, pp. 66-83, 2017.

[43] G. Zong, D. Yang, L. Hou et al., "Robust finite-time $H_{\infty}$ control for Markovian jump systems with partially known transition probabilities," Journal of the Franklin Institute, vol. 350, no. 6, pp. 1562-1578, 2013.

[44] H. Ren and G. Zong, "Robust input-output finite-time filtering for uncertain Markovian jump nonlinear systems with partially known transition probabilities," International Journal of Adaptive Control and Signal Processing, vol. 31, no. 10, pp. 1437-1455, 2017.

[45] Y. Zhang, W. Liu, G. Wang et al., "Stabilization of discretetime Markovian jump systems with partially unknown transition probabilities," Discrete and Continuous Dynamical Systems-Series B, vol. 16, no. 4, pp. 1197-1211, 2017.

[46] O. Solomon and E. Fridman, "Stability and passivity analysis of semilinear diffusion PDEs with time-delays," International Journal of Control, vol. 88, no. 1, pp. 180-192, 2015.
[47] E. Vesentini, "Semigroups of linear operators and applications to partial differential equations," Applied Mathematical Sciences, vol. 44, no. 3, pp. 343-369, 1983.

[48] H. L. Xing, D. H. Li, and W. S. Cui, "Robust control guaranteeing uniform ultimate boundedness for a class of mismatched uncertain parabolic distributed parameter systems," International Journal of Control, Automation and Systems, vol. 11, no. 4, pp. 728-733, 2013.

[49] Y. P. Luo, W. H. Xia, G. R. Liu et al., "LMI approach to exponential stabilization of distributed parameter control systems with delay," Acta Automatica Sinica, vol. 35, no. 3, pp. 299-304, 2009.

[50] E. Fridman and Y. Orlov, "Exponential stability of linear distributed parameter systems with time-varying delays," Automatica, vol. 45, no. 1, pp. 194-201, 2009.

[51] H. L. Xing, C. C. Gao, G. Y. Tang et al., "Variable structure sliding mode control for a class of uncertain distributed parameter systems with time-varying delays," International Journal of Control, vol. 82, no. 2, pp. 287-297, 2009.

[52] C. C. Gao, Z. Liu, and R. P. Xu, "Feedback control for a class of distributed parameter systems with continuous distributed time delay," Control and Decision, vol. 28, no. 3, pp. 446-450, 2013.

[53] B. T. Cui and X. Y. Lou, Theory and Its Application for Distributed Parameter Systems with Time-Delays, National Defense Industry Press, Beijing, China, 2009.

[54] Z. C. Chen, Partial Differential Equation, China University of Science and Technology Press, Hefei, China, 2002.

[55] E. N. Sanchez and J. P. Perez, "Input-to-state stability analysis for dynamic neural network," IEEE Transactions on Circuits and Systems I: Fundamental Theory and Applications, vol. 46, no. 11, pp. 1395-1398, 1999. 